\title{
Loop Gain Measurement of Paralleled DC-DC Converters With Average-Current-Sharing Control
}

\author{
Yuri Panov and Milan M. Jovanović, Fellow, IEEE
}

\begin{abstract}
Paralleled operation of dc-de converters is widely used in today's distributed power systems. Special control circuitry is usually necessary to ensure current sharing among the paralleled modules. The average-current-sharing control method is very popular due to its simple implementation and good performance. Although the analysis and design of the average-current-sharing control were presented in the past, a loop gain measurement technique has not been published. This paper presents a practical loop gain measurement technique that uses two excitation sources. Measured and theoretical data are compared to verify the proposed technique.
\end{abstract}

Index Terms-Average-current-sharing control, current sharing, current-sharing loop gain, loop gain measurement, paralleled dc-dc converters.

\section{INTRODUCTION}

$\mathbf{P}$ ARALLEL operation of dc-dc converters is widely used in today's distributed power systems due to redundancy, expandability of output power, easy standardization, and improved thermal management. Generally, it is desirable to distribute the load current equally among paralleled converters. However, because of limited component tolerances and an asymmetric layout of the converters, their output currents can be significantly different. To balance the load current among the paralleled modules, a variety of approaches, with different complexities and current-sharing performances, were proposed and employed by industry [1]-[6].

The simplest current-sharing technique is the open-loop (droop) method [1]-[3], which relies on the output resistance of paralleled modules to maintain relatively even current distribution among the modules. The droop method is easy to implement and it does not require any communication (control wire connection) between the modules. However, this technique has poor output voltage regulation as a result of droop output characteristics and requires individual converter reference voltages to be trimmed to closely match each other.

More complex, closed-loop (active) current-sharing techniques [3]-[10] overcome the disadvantage of poor output regulation by employing feedback control. One of the most popular

Manuscript received April 24, 2008; revised July 1, 2008; accepted July 4, 2008. Current version published December 9, 2008. Recommended for publication by Associate Editor Y.-F. Liu. This paper was presented in part at the Twenty-Third Annual IEEE Applied Power Electronics Conference (APEC), Austin, TX, February 24-28, 2008.

The authors are with Delta Products Corporation, Power Electronics Laboratory, Research Triangle Park (RTP), NC 27709 USA (e-mail: ypanov@deltartp.com).

Color versions of one or more of the figures in this paper are available online at http://ieeexplore.ieee.org.

Digital Object Identifier 10.1109/TPEL.2008.2002955

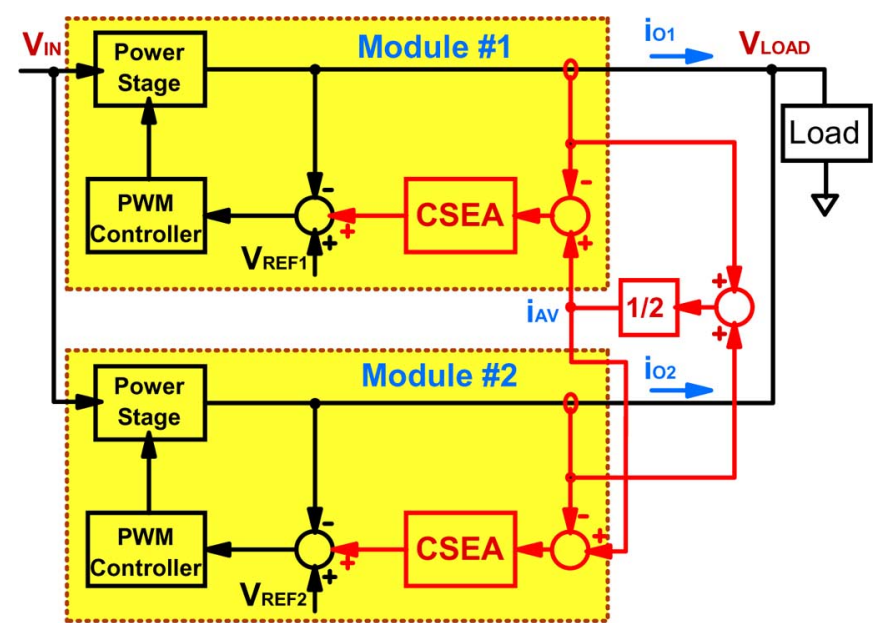

Fig. 1. Simplified block diagram of paralleled converters with averagecurrent-sharing control.

closed-loop methods is an average-current-sharing method [10] due to its simple implementation and high performance. A simplified functional diagram of paralleled converters with averagecurrent-sharing control is shown in Fig. 1.

The output currents are sensed and compared to average converter output current $i_{\mathrm{AV}}$. The resulting current-sharing error signals are then processed by current-sharing-error amplifiers (CSEAs) and injected in the voltage loops of paralleled modules. When the CSEA gain is high, the corresponding currentsharing error is low, and currents of the paralleled modules are essentially equal.

The steady-state performance of the average-current-sharingcontrol technique was analyzed in [9]. For paralleled converters delivering power to modern data processing equipment, the dynamic current-sharing performance during fast-slew-rate load transients becomes more and more important. To provide adequate transient current sharing, the high bandwidth of the current-sharing loop as well as ample stability margins are required. The dynamic modeling and analysis of average-currentsharing control can be found in [4] and [11]-[14]. Perhaps the most general and systematic analysis was presented in [12] and [13]. Although the references contain dynamic modeling, analysis, and design of average-current-sharing control, none of them offers a method of measuring the current-sharing loop gain in the hardware setup. The purpose of this paper is to propose a practical technique for measurement of the average-currentsharing loop gain, derive the theoretical loop gain, and compare the measured and calculated results. 


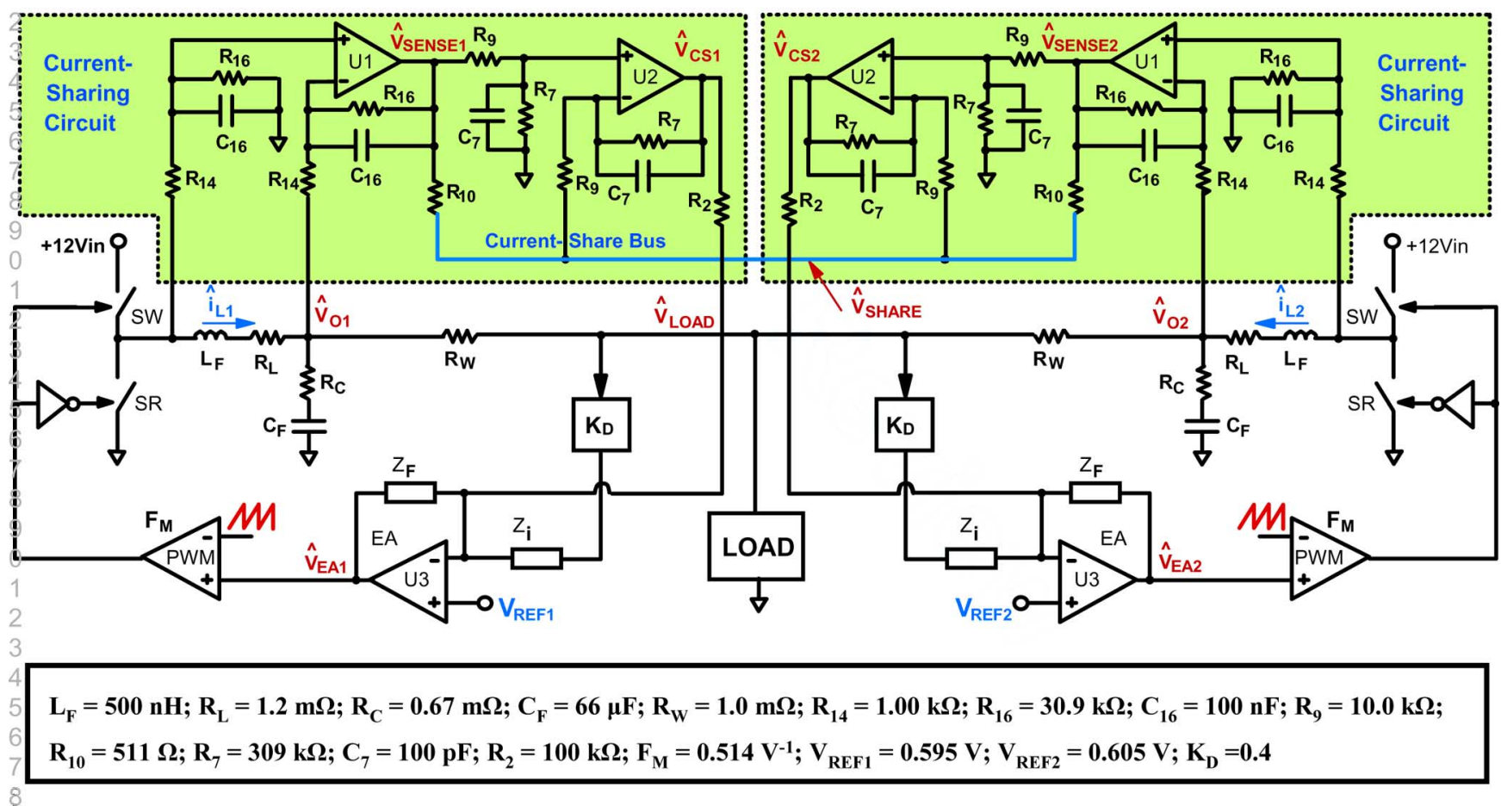

Fig. 2. Simplified circuit diagram of paralleled POL converters and list of component values.

\section{Current-Sharing Modeling AND CAlCUlated LoOP GAIN OF PARALLELED DC-DC CONVERTERS}

A detailed schematic of two paralleled buck converter modules with synchronous rectifiers (SRs), which employ the average-current-sharing control, and the list of component values are shown in Fig. 2. Blocks $K_{D}$ represent the gain of load voltage resistive dividers and resistors $R_{W}$ represent the interconnect impedance. The modules in Fig. 2 are identical except for mismatched reference voltages $V_{\mathrm{REF} 1}$ and $V_{\mathrm{REF} 2}$. Current balancing of modules' output currents is achieved through balancing of their inductor currents $i_{L 1}$ and $i_{L 2}$. Balancing of the inductor currents has the advantage of lossless current sensing [15]. Namely, the module inductor current is sensed based on the voltage drop across equivalent series resistance (ESR) $R_{L}$ of inductor $L_{F}$. The voltage across inductor $L_{F}$ is amplified and averaged by differential amplifier U1, whose transfer function has a low-pass filter property. Voltage $\hat{V}_{\text {SENSE }}$ at the output of amplifier U1 is related to inductor current $\hat{\boldsymbol{i}}_{L}$ as

$$
\hat{\boldsymbol{V}}_{\mathrm{SENSE}}=\hat{\boldsymbol{i}}_{L} \boldsymbol{Z}_{X}
$$

where $\boldsymbol{Z}_{X}=\boldsymbol{R}_{16} / \boldsymbol{R}_{14} \cdot\left(\boldsymbol{R}_{L}+\mathbf{s} \cdot \boldsymbol{L}_{F}\right) /\left(1+\mathbf{s} \cdot \boldsymbol{R}_{16} \cdot \boldsymbol{C}_{16}\right)$.

Voltage $\hat{\boldsymbol{V}}_{\text {SHARE }}$ on the current-share bus is the average of signals $\hat{\boldsymbol{V}}_{\mathrm{SENSE} 1}$ and $\hat{\boldsymbol{V}}_{\mathrm{SENSE} 2}$

$$
\hat{\boldsymbol{V}}_{\mathrm{SHARE}}=\frac{1}{2}\left(\hat{\boldsymbol{V}}_{\mathrm{SENSE} 1}+\hat{\boldsymbol{V}}_{\mathrm{SENSE} 2}\right) .
$$

The difference between sensed voltage $\hat{V}_{\text {SENSE }}$ and currentshare bus voltage $\hat{\boldsymbol{V}}_{\mathrm{SHARE}}$ is proportional to the current-sharing error. This error is amplified by differential amplifier U2, which has a transfer function $\boldsymbol{G}_{\mathrm{CS}}=\left(\boldsymbol{R}_{7} / \boldsymbol{R}_{9}\right) /\left(\mathbf{1}+\mathbf{s} \cdot \boldsymbol{R}_{7} \cdot \boldsymbol{C}_{7}\right)$.
Resistor $R 2$ at the output of amplifier U2 converts voltage $\hat{\boldsymbol{V}}_{\mathrm{CS}}$ into current, which is injected into the voltage loop, as shown in Fig. 2. Current-sharing signals $\hat{\boldsymbol{V}}_{\mathrm{CS} 1}$ and $\hat{\boldsymbol{V}}_{\mathrm{CS} 2}$, which inject currents in the voltage loops, are derived as

$$
\begin{aligned}
& \hat{\boldsymbol{V}}_{\mathrm{CS} 1}=\boldsymbol{G}_{\mathrm{CS}} \frac{\left(\hat{\boldsymbol{V}}_{\mathrm{SENSE} 1}-\hat{\boldsymbol{V}}_{\mathrm{SENSE} 2)}\right.}{2} \\
& \hat{\boldsymbol{V}}_{\mathrm{CS} 2}=\boldsymbol{G}_{\mathrm{CS}} \frac{\left(\hat{\boldsymbol{V}}_{\mathrm{SENSE} 2}-\hat{\boldsymbol{V}}_{\mathrm{SENSE} 1)}\right.}{2}=-\hat{\boldsymbol{V}}_{\mathrm{CS} 1} .
\end{aligned}
$$

Equation (3) indicates that signals $\hat{\boldsymbol{V}}_{\mathrm{CS} 1}$ and $\hat{\boldsymbol{V}}_{\mathrm{CS} 2}$ are equal in magnitude, but opposite in phase. Error amplifier U3 of the voltage feedback loop is described by

$$
\hat{\boldsymbol{V}}_{\mathrm{EA}}=-\boldsymbol{G}_{\mathrm{EA} 1} \hat{\boldsymbol{V}}_{\mathrm{LOAD}}-\boldsymbol{G}_{\mathrm{EA} 2} \hat{\boldsymbol{V}}_{\mathrm{CS}}
$$

where $\boldsymbol{G}_{\mathrm{EA} 1}=\boldsymbol{Z}_{F} / \boldsymbol{Z}_{i}$ and $G_{\mathrm{EA} 2}=\boldsymbol{Z}_{F} / \boldsymbol{R}_{2}$.

The averaged model of two combined power stages is shown in Fig. 3. Resistors $R_{\mathrm{LOSS}}$ in Fig. 3 model conduction losses of the switch and SR. The combined power stage is described by

$$
\begin{aligned}
\hat{\boldsymbol{V}}_{O 1} & =\boldsymbol{G}_{v d} \hat{\boldsymbol{d}}_{1}+\boldsymbol{G}_{v d}^{c c} \hat{\boldsymbol{d}}_{2} \quad \hat{\boldsymbol{V}}_{O 2}=\boldsymbol{G}_{v d}^{c c} \hat{\boldsymbol{d}}_{1}+\boldsymbol{G}_{v d} \hat{\boldsymbol{d}}_{2} \\
\hat{\boldsymbol{i}}_{L 1} & =\boldsymbol{G}_{i d} \hat{\boldsymbol{d}}_{1}+\boldsymbol{G}_{i d}^{c c} \hat{\boldsymbol{d}}_{2} \quad \hat{\boldsymbol{i}}_{L 2}=\boldsymbol{G}_{i d}^{c c} \hat{\boldsymbol{d}}_{1}+\boldsymbol{G}_{i d} \hat{\boldsymbol{d}}_{2} \\
\hat{\boldsymbol{V}}_{\mathrm{LOAD}} & =\frac{\boldsymbol{G}_{v d}+\boldsymbol{G}_{v d}^{c c}}{\boldsymbol{2}+\boldsymbol{R}_{W} / \boldsymbol{Z}_{\mathrm{LOAD}}} \cdot\left(\hat{\boldsymbol{d}}_{1}+\hat{\boldsymbol{d}}_{2}\right)
\end{aligned}
$$

where $\boldsymbol{G}_{v d}=\hat{\boldsymbol{V}}_{O 1} / \hat{\boldsymbol{d}}_{1}=\hat{\boldsymbol{V}}_{O 2} / \hat{\boldsymbol{d}}_{2}, \boldsymbol{G}_{i d}=\hat{\boldsymbol{i}}_{L 1} / \hat{\boldsymbol{d}}_{1}=\hat{\boldsymbol{i}}_{L 2} / \hat{\boldsymbol{d}}_{2}$ are direct transfer functions, and $\boldsymbol{G}_{v d}^{c c}=\hat{\boldsymbol{V}}_{O 2} / \hat{\boldsymbol{d}}_{1}=\hat{\boldsymbol{V}}_{O 1} / \hat{\boldsymbol{d}}_{2}$, $\boldsymbol{G}_{i d}^{c c}=\hat{\boldsymbol{i}}_{L 1} / \hat{\boldsymbol{d}}_{2}=\hat{\boldsymbol{i}}_{L 2} / \hat{\boldsymbol{d}}_{1}$ are cross-coupling transfer functions. 


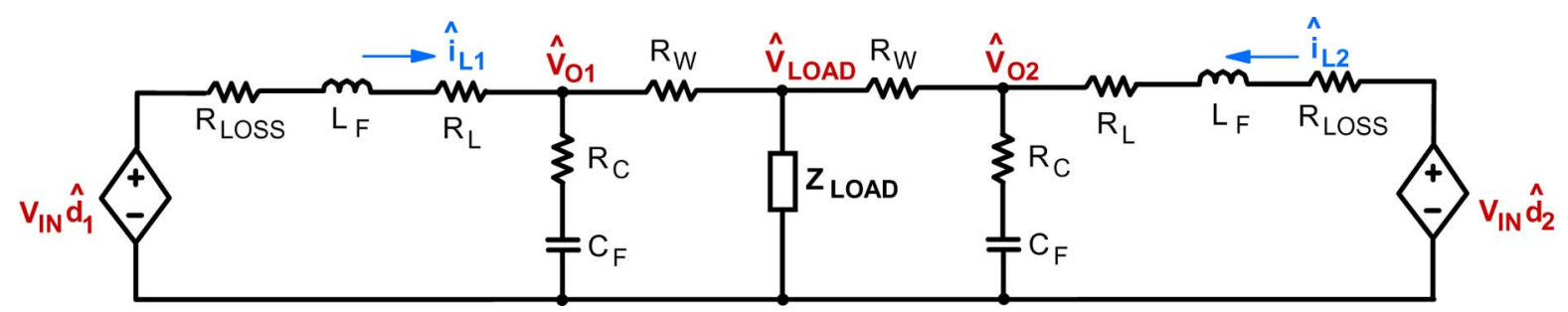

Fig. 3. Averaged model of merged power stages of two paralleled modules.

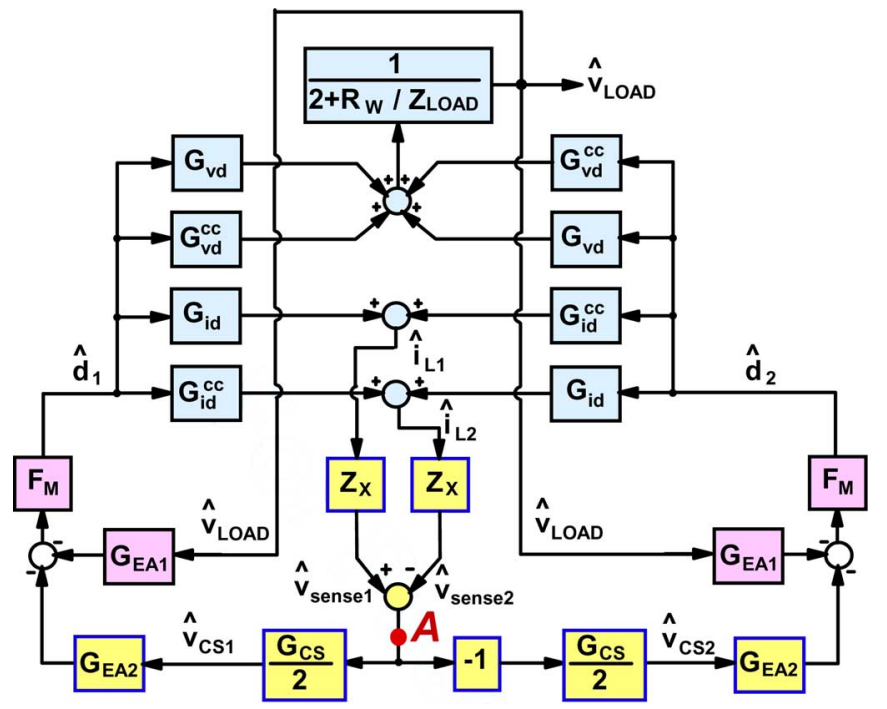

Fig. 4. Block diagram of two paralleled modules.

Based on (1)-(7), the control block diagram of paralleled modules with remote voltage sensing is shown in Fig. 4. Since the diagram is symmetrical and $\hat{\boldsymbol{V}}_{\mathrm{CS} 1}=-\hat{\boldsymbol{V}}_{\mathrm{CS} 2}$, duty cycles $\hat{\boldsymbol{d}}_{1}$ and $\hat{\boldsymbol{d}}_{2}$ are also equal in magnitude and opposite in phase, namely, $\hat{\boldsymbol{d}}_{1}=-\hat{\boldsymbol{d}}_{2}$. Based on (6) and (7)

$$
\hat{\boldsymbol{V}}_{\mathrm{LOAD}}=0 \quad \text { and } \quad \hat{\boldsymbol{i}}_{L 1}=-\hat{\boldsymbol{i}}_{L 2} .
$$

Relationships (8) allow reduction of the block diagram, as shown in Fig. 5. Since $\hat{V}_{\text {LOAD }}=0$, signals excited by sources $\boldsymbol{V}_{\mathrm{IN}} \hat{\boldsymbol{d}}_{1}$ and $\boldsymbol{V}_{\mathrm{IN}} \hat{\boldsymbol{d}}_{2}$ cannot propagate to the power stages of the opposite modules, and therefore, $\boldsymbol{G}_{i d}^{c c}=0$ and $\boldsymbol{G}_{v d}^{c c}=0$.

Based on the reduced block diagram in Fig. 5, the currentsharing loop gain is finally derived as

$$
\boldsymbol{T}_{\mathrm{CS}}=\boldsymbol{G}_{\mathrm{EA} 2} \boldsymbol{F}_{M} \boldsymbol{G}_{i d} \boldsymbol{Z}_{X} \boldsymbol{G}_{\mathrm{CS}}
$$

It should be noted that power stage transfer function $\boldsymbol{G}_{i d}=$ $\hat{\boldsymbol{i}}_{L 1} / \hat{\boldsymbol{d}}_{1}$ in (9) is calculated with the shorted load since $\hat{\boldsymbol{V}}_{\text {LOAD }}=$ 0 . This condition also means that current-sharing loop gain $T_{\mathrm{CS}}$ is independent of the load.

Derived loop gain $T_{C S}$ is practical since it provides the basis for conventional design of current-sharing error amplifier transfer function $\boldsymbol{G}_{\mathrm{CS}}$ in the frequency domain.

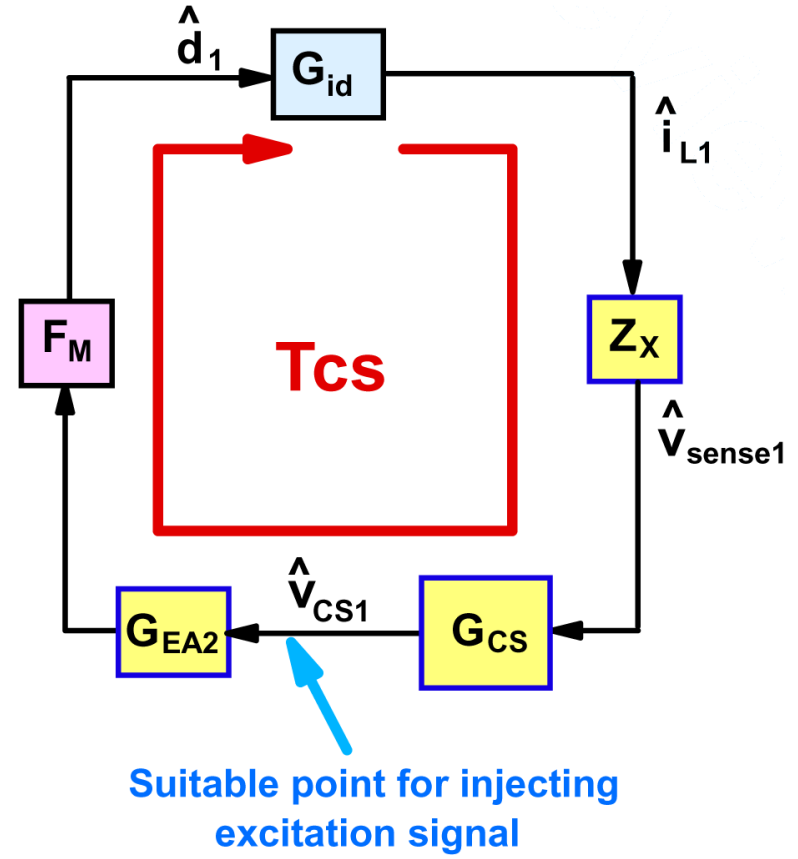

Fig. 5. Reduced block diagram for loop gain derivation.

\section{PRoposed CURRENT-Sharing LOOP GAIN HARdWARE MEASUREMENT}

Generally, to measure a meaningful current-sharing loop gain, it is desirable to identify the location where breaking the loop interrupts the flow of current-sharing signals of both modules. At that location, the excitation signal is added and the loop gain is measured as the ratio of the test and reference signals. Observation of the diagram in Fig. 4 suggests that the only location that satisfies the indicated condition is location A. If the excitation signal is added at location $\mathbf{A}$, then $\hat{\boldsymbol{V}}_{\mathrm{CS} 1}=-\hat{\boldsymbol{V}}_{\mathrm{CS} 2}$, $\hat{\boldsymbol{d}}_{1}=-\hat{\boldsymbol{d}}_{2}, \hat{\boldsymbol{V}}_{\mathrm{LOAD}}=0, \hat{\boldsymbol{i}}_{L 1}=-\hat{\boldsymbol{i}}_{L 2}$, i.e., the signal relationships of the original circuit are observed. Unfortunately, point A exists only on the block diagram and not in the real circuit. Therefore, a different measurement approach should be considered. To derive the desired measurement method, the block diagram in Fig. 6 is examined. The excitation source can be added at the output of amplifier U2 in Fig. 2 where the relationship between the input and output impedances is maintained [16], [17]. Specifically, the magnitude of U2 output impedance is much less than the value of resistor R2. If the single injection source $\hat{\boldsymbol{V}}_{\mathrm{ac} 1}$ is added, as shown in Fig. 6, then $\hat{\boldsymbol{d}}_{1} \propto \hat{\boldsymbol{V}}_{\mathrm{CS} 1}+\hat{\boldsymbol{V}}_{\mathrm{ac}}$ and $\hat{\boldsymbol{d}}_{2} \propto \hat{\boldsymbol{V}}_{\mathrm{CS} 2} \propto-\hat{\boldsymbol{V}}_{\mathrm{CS} 1}$. Hence, $\hat{\boldsymbol{d}}_{2} \neq-\hat{\boldsymbol{d}}_{1}$ and the signal 


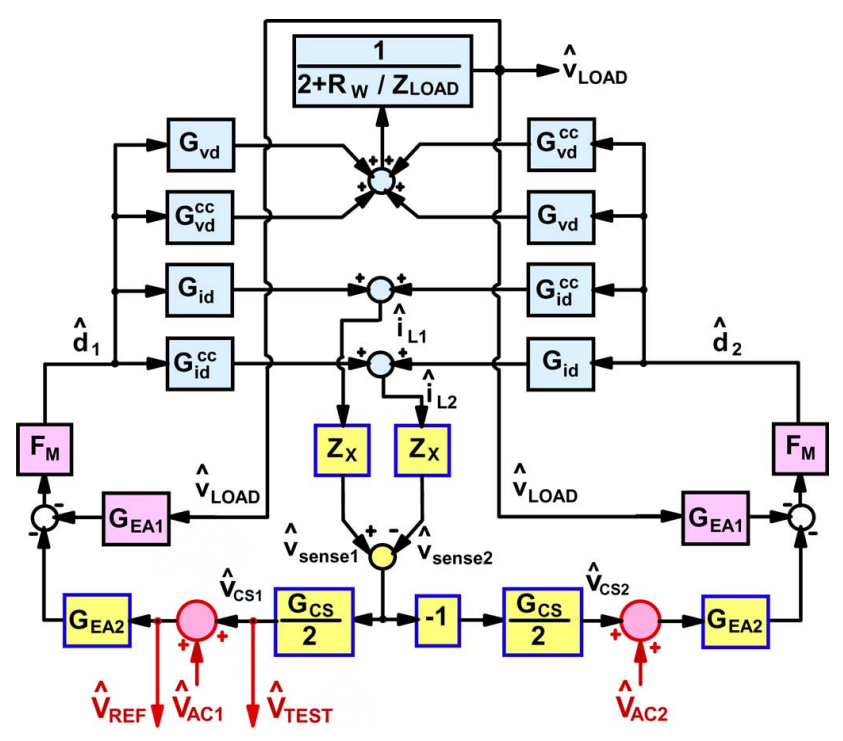

Fig. 6. Placement of excitation sources for loop gain measurement.
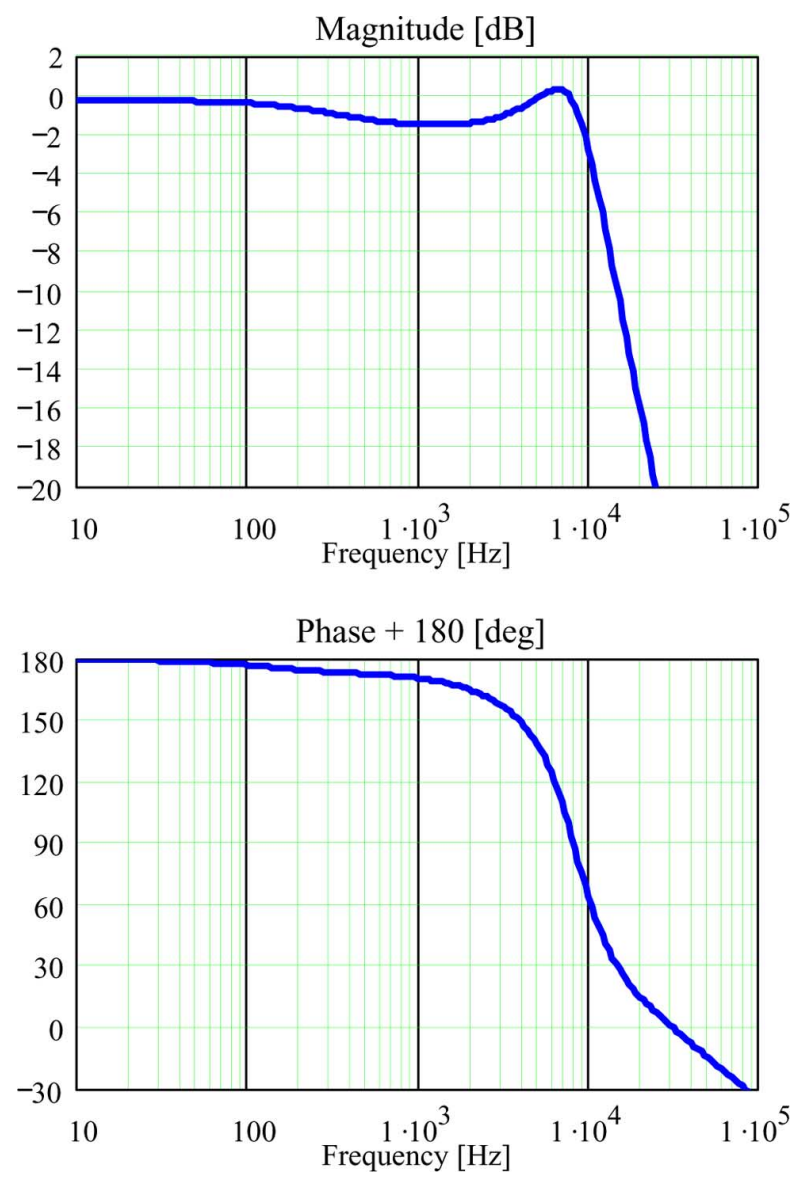

Fig. 7. Simulated Bode plots of current-sharing loop gain $\boldsymbol{T}_{\mathrm{CS} 1}$ with singleinjection source.

balance of the original circuit is violated. To demonstrate the drawbacks of the single-injection-source measurement, corresponding loop gain $T_{\mathrm{CS} 1}$ was simulated using SIMPLIS software for paralleled converters, shown in Fig. 2 and plotted in Fig. 7. Loop gain $T_{\mathrm{CS} 1}$ stays below $0 \mathrm{~dB}$ level at low frequencies and goes above $0 \mathrm{~dB}$ in the frequency range from 5.3 to $7.6 \mathrm{kHz}$.

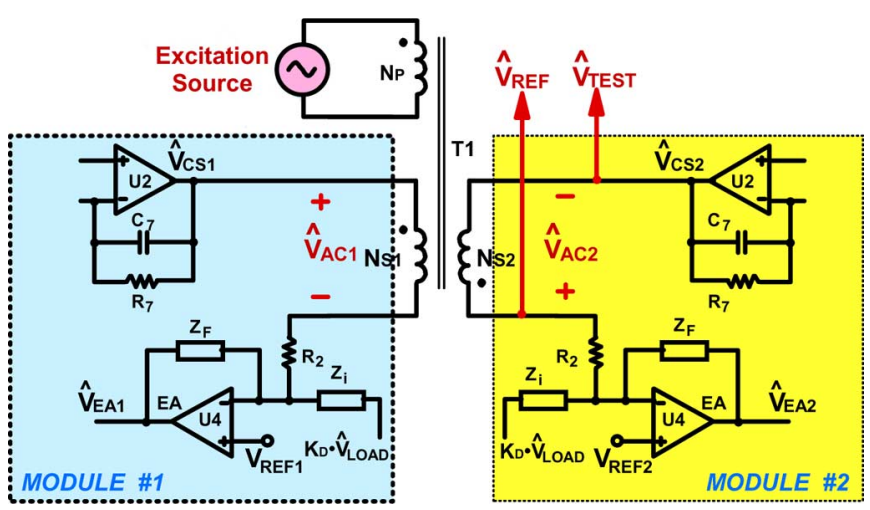

Fig. 8. Practical setup for current sharing loop gain measurement.

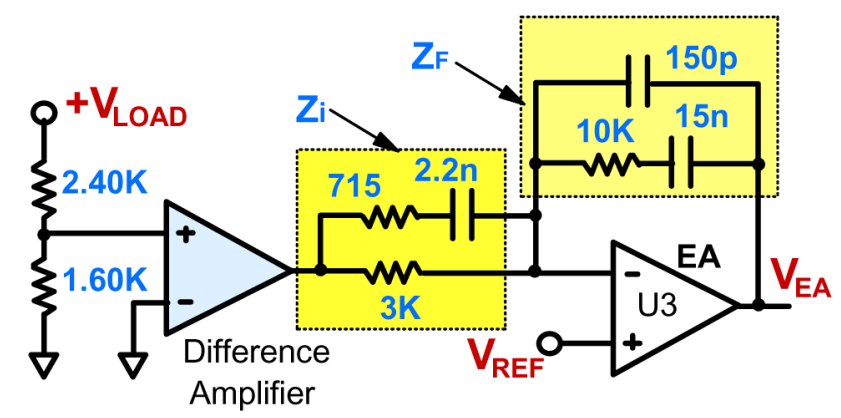

Fig. 9. Implementation of load voltage sensing circuit and EA.

The maximum value of this gain in this range is only $0.3 \mathrm{~dB}$. Above $7.6-\mathrm{kHz}$ frequency, loop gain $\boldsymbol{T}_{\mathrm{CS} 1}$ decreases rapidly with $-40 \mathrm{~dB} /$ decade slope. This loop gain still carries information about the current-sharing loop stability. Namely, gain $\boldsymbol{T}_{\mathrm{CS} 1}$ indicates that the loop is stable with the minimum phase margin of $100^{\circ}$, which corresponds to $7.6-\mathrm{kHz}$ crossover frequency, and with the gain margin above $20 \mathrm{~dB}$. However, it is not possible to define a meaningful bandwidth for loop gain $\boldsymbol{T}_{\mathrm{CS} 1}$. Also, the traditional design of current-sharing error amplifier compensation in the frequency domain is not possible since there are two current-sharing amplifiers in the circuit and relationship between loop gain $\boldsymbol{T}_{\mathrm{CS} 1}$ and current-sharing-amplifier transfer function $\boldsymbol{G}_{\mathrm{CS}}$ is not straightforward. Hence, the single-sourceinjection measurement technique fails and a more sophisticated technique should be proposed.

In order to keep the signal balance and measure meaningful loop gain $T_{\mathrm{CS}}$, two excitation sources $\hat{\boldsymbol{V}}_{\text {ac1 }}$ and $\hat{\boldsymbol{V}}_{\text {ac2 }}$ are added, as shown in Fig. 6. In this case, $\hat{\boldsymbol{d}}_{1} \propto \hat{\boldsymbol{V}}_{\mathrm{CS} 1}+\hat{\boldsymbol{V}}_{\mathrm{ac} 1}$ and $\hat{\boldsymbol{d}}_{2} \propto-\hat{\boldsymbol{V}}_{\mathrm{CS} 2}+\hat{\boldsymbol{V}}_{\mathrm{ac} 2} \propto-\hat{\boldsymbol{V}}_{\mathrm{CS} 1}+\hat{\boldsymbol{V}}_{\mathrm{ac} 2}$. If excitation sources have the same magnitude and the opposite phase, i.e., $\hat{\boldsymbol{V}}_{\text {ac2 }}=$ $-\hat{\boldsymbol{V}}_{\mathrm{ac} 1}$, then $\hat{\boldsymbol{d}}_{2}=-\hat{\boldsymbol{d}}_{1}, \hat{\boldsymbol{V}}_{\mathrm{LOAD}}=0, \hat{\boldsymbol{i}}_{L 1}=-\hat{\boldsymbol{i}}_{L 2}$, and the signal balance of the original circuit is restored. It is easy to verify that with two excitation sources the loop gain is the same as the one given by (9). Therefore, the current-sharing loop gain measurement can be accomplished with two excitation sources $\hat{\boldsymbol{V}}_{\mathrm{ac} 1}$ and $\hat{\boldsymbol{V}}_{\mathrm{ac} 2}=-\hat{\boldsymbol{V}}_{\mathrm{ac} 1}$. The two sources can be implemented as voltages of the opposite polarity on two secondary windings of a three-winding excitation transformer. The secondary windings should have the same number of turns $\boldsymbol{N}_{S 1}=\boldsymbol{N}_{S 2}$. A 
Magnitude [dB]

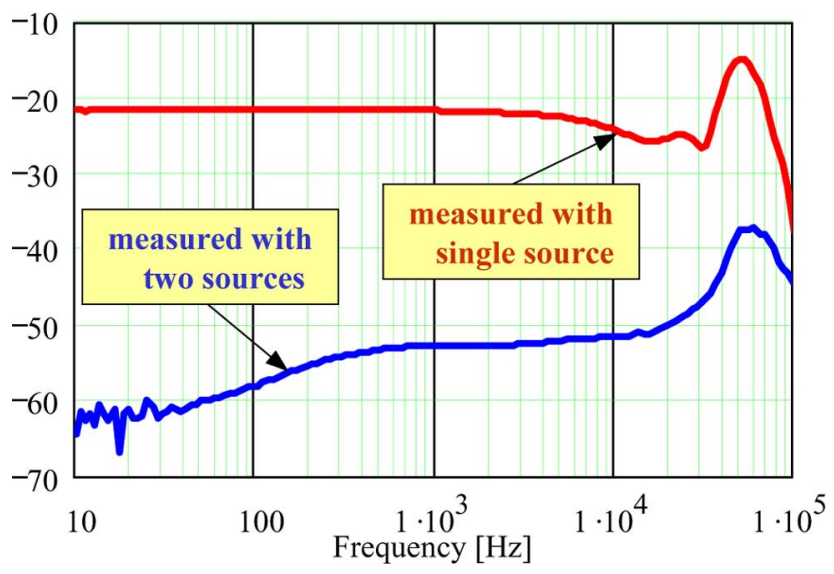

Fig. 10. Measured transfer function $\hat{\boldsymbol{V}}_{\mathrm{LOAD}} / \hat{\boldsymbol{V}}_{\mathrm{CS}}$.
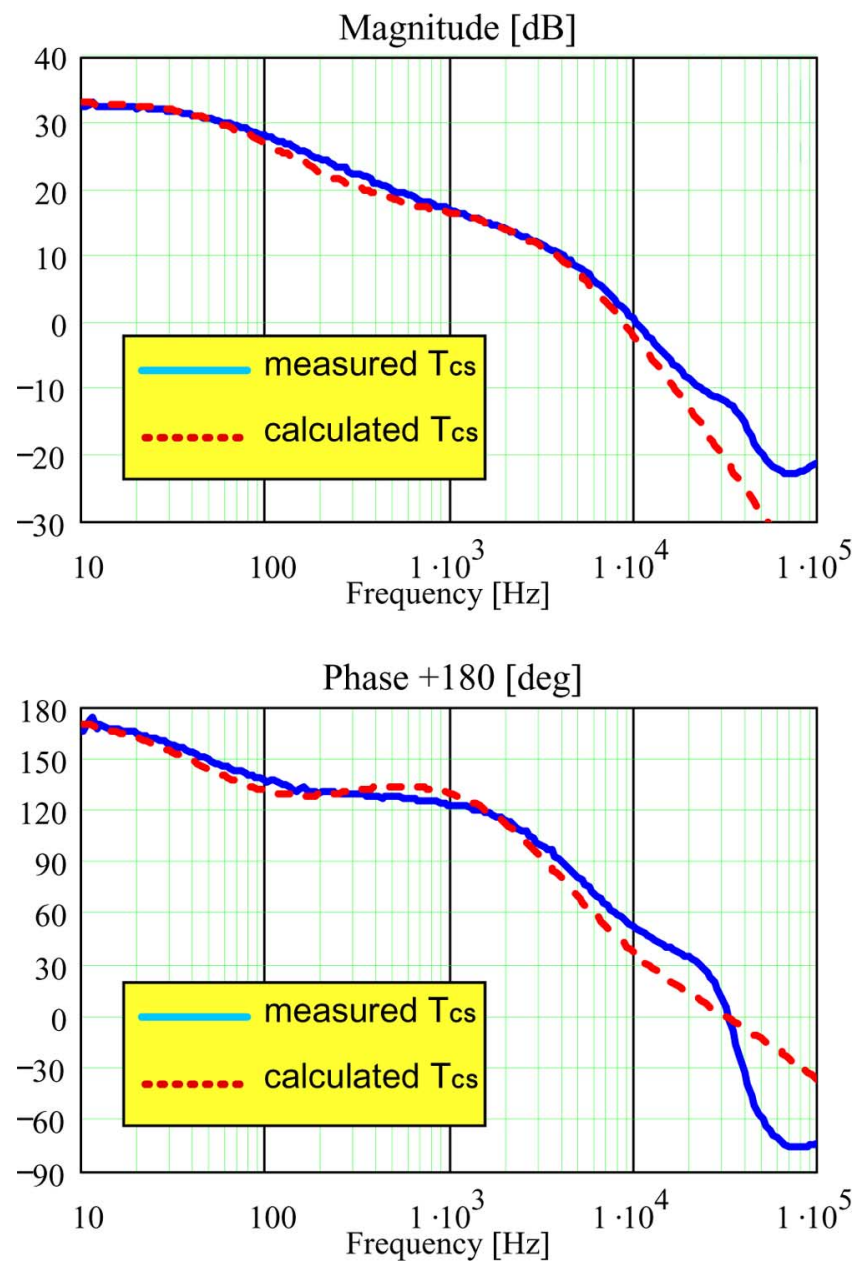

Fig. 11. Measured and calculated loop gain $\boldsymbol{T}_{\mathrm{CS}}$.

practical measurement setup is shown in Fig. 8. The currentsharing loop gain is then measured as the ratio $\hat{\boldsymbol{V}}_{\mathrm{TEST}} / \hat{\boldsymbol{V}}_{\mathrm{REF}}$. Since the paralleled modules are identical, the pair of $\hat{\boldsymbol{V}}_{\mathrm{REF}}$ and $\hat{\boldsymbol{V}}_{\text {TEST }}$ signals can be measured on either module.
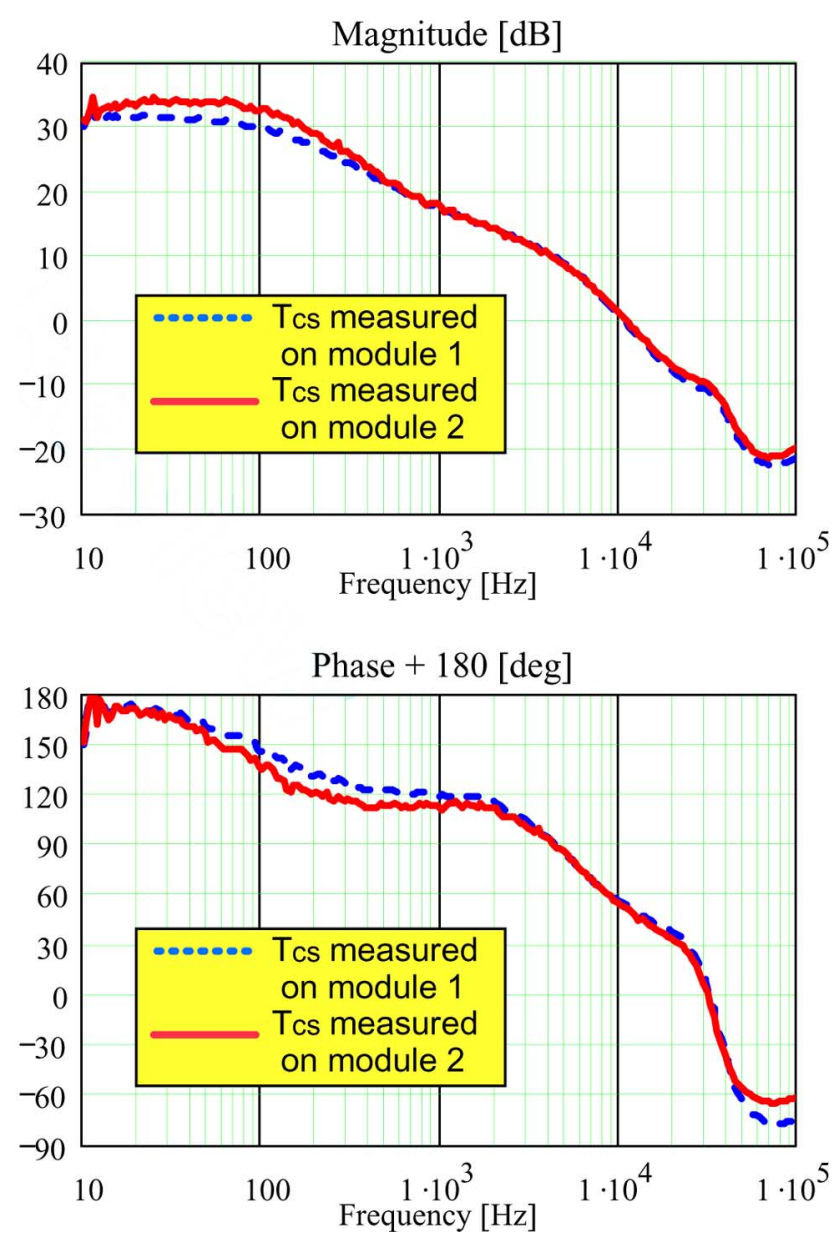

Fig. 12. Current-sharing loop gain $\boldsymbol{T}_{\mathrm{CS}}$ measured on both paralleled modules.

\section{EXPERIMENTAL RESULTS}

To test the proposed measurement technique, two $330-\mathrm{kHz}$, 12-V input, 20-A output point-of-load (POL) converters with adjustable output voltage were paralleled. The simplified schematic and component values of the POL converters are shown in Fig. 2. Measurements of transfer functions were conducted at $1.5-\mathrm{V}$ output voltage and $40-\mathrm{A}$ load current. A threewinding transformer for the signal injection was implemented as follows. The transformer has Ferroxcube PTS30/19/I-3C90 ferrite core and three identical windings of 160 turns. Solid copper wire of American Wire Gauge (AWG) 30 is used for the windings, which are bifilar wound. The transformer has a high magnetizing inductance of $68 \mathrm{mH}$, and a low leakage inductance of $23 \mu \mathrm{H}$, allowing measurement in a wide frequency range from $10 \mathrm{~Hz}$ up to several hundred $\mathrm{kHz}$.

The implementation of the voltage-loop error amplifier, which was used in the experimental setup, is shown in Fig. 9. The difference amplifier in Fig. 9 provides differential sensing of the remote load voltage.

Before measuring the loop gain, the assumption of zero perturbation of the load voltage, used in Sections II and III, has to be verified. Since the real modules are not identical due to component tolerances and asymmetrical layout of the system 

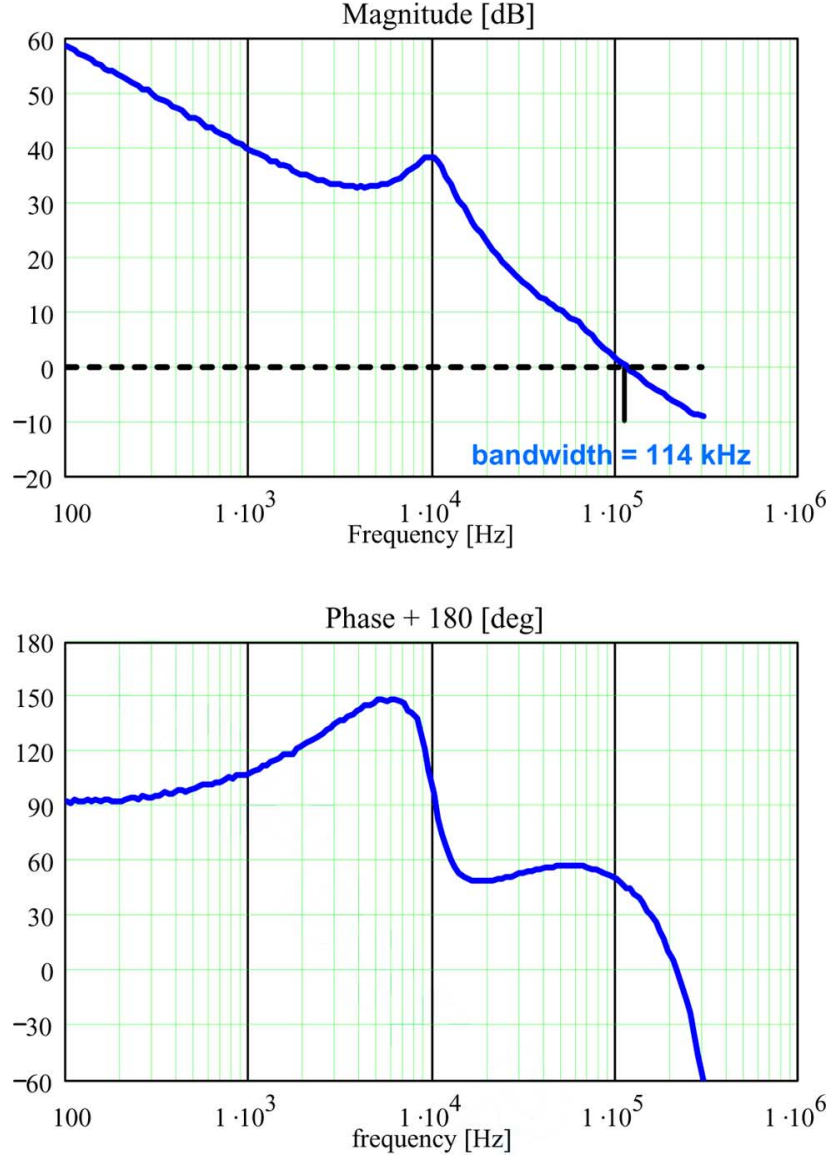

Fig. 13. Measured voltage loop gain of stand-alone module.

board, the load voltage perturbation cannot be exactly zero. To verify this assumption of the proposed measurement technique, transfer function $\hat{\boldsymbol{V}}_{\mathrm{LOAD}} / \hat{\boldsymbol{V}}_{\mathrm{CS}}$ was measured in setups with one and two excitation sources. The measured results for component values, shown in Figs. 2 and 9, can be seen in Fig. 10. Fig. 10 demonstrates that the two-source injection dramatically reduces ac component of $V_{\mathrm{LOAD}}$ and makes it practically zero in the frequency range of interest. The measured and calculated current-sharing loop gains are plotted in Fig. 11 and show reasonable agreement. The measured loop gain has $10.4-\mathrm{kHz}$ bandwidth, $51^{\circ}$ phase margin, and $13-\mathrm{dB}$ gain margin, whereas the calculated loop gain has $8.6-\mathrm{kHz}$ bandwidth, $44^{\circ}$ phase margin, and 21-dB gain margin. The measured and calculated loop gains start to diverge at high frequencies where the load voltage perturbation signal increases significantly.

In the setup with two excitation sources, loop gain $\boldsymbol{T}_{\mathrm{CS}}$ can be measured as the ratio of signals on the sides of either source. Fig. 12 shows the current-sharing loop gains measured on the sides of both sources. The Bode plots in Fig. 12 are not identical due to component tolerances of the paralleled modules, but match closely.

The measured voltage-loop gain of the stand-alone experimental module is plotted in Fig. 13. The measurement was conducted with five Panasonic polymer aluminum $150-\mu \mathrm{F}, 12-$ $\mathrm{m} \Omega$ ESR load capacitors. Since the voltage loop gain in Fig. 13 has 114-kHz bandwidth, there is sufficient separation between gain crossover frequencies of the voltage and current-sharing loops to prevent any undesirable interaction.

\section{SUMMARY}

Sufficient bandwidth and stability margins of the currentsharing loop are important for the design of high-performance paralleled $\mathrm{dc}-\mathrm{dc}$ converters. Measurement of a meaningful current-sharing loop gain cannot be accomplished with traditional single-injection-source approach. To measure the currentsharing loop gain, a setup with two injection sources of the same magnitude and opposite phase was proposed. Two-source injection is easily implemented by using an excitation transformer with two secondary windings that have the same number of turns. The current-sharing loop gain was derived for identical converters with mismatched references and was compared to the measured loop gain of two paralleled POL converters. Good agreement between the measured and calculated current-sharing loop gains verifies the proposed measurement technique.

\section{REFERENCES}

[1] B. Irving and M. Jovanovic, "Analysis, design and performance evaluation of droop current-sharing method," in Proc. IEEE Appl. Power Electron. Conf., Feb. 2000, pp. 235-241.

[2] C. Jamerson and C. Mullett, "Paralleling supplies via various droop methods," in Proc. High-Freq. Power Convers. Conf., 1994, pp. 68-76.

[3] J. W. Kim, H. S. Choi, and B. H. Cho, "A novel droop method for converter parallel operation," IEEE Trans. Power Electron., vol. 17, no. 1, pp. 25 32, Jan. 2002.

[4] S. Luo, Z. Ye, R.-L. Lin, and F. C. Lee, "A classification and evaluation of paralleling methods for power supply modules," in Proc. IEEE Power Electron. Spec. Conf. (PESC), 1999, pp. 901-908.

[5] J. Abu-Qahouq, H. Mao, and I. Batarseh, "Multiphase voltage-mode hysteretic controlled dc-dc converter with novel current sharing," IEEE Trans. Power Electron., vol. 19, no. 6, pp. 1397-1162, Nov. 2004.

[6] J. J. Shieh, "Peak current-mode based single-wire current-share multimodule paralleling dc power supplies," IEEE Trans. Circuits Syst. I, Fundam. Theory Appl., vol. 50, no. 12, pp. 1564-1568, Dec. 2003.

[7] P. Li and B. Lehman, "A design method for paralleling current mode controlled dc-dc converters," IEEE Trans. Power Electron., vol. 19, no. 3, pp. 748-756, May 2004.

[8] M. Jordan, "UC3907 load share IC simplifies parallel power supply design," Texas Instruments Application Note U-129, Dallas, TX.

[9] L. Balogh, "Paralleling power-Choosing and applying the best technique for load sharing," in Proc. Texas Instrum. Power Design Seminar, 2002, pp. 1-6-30.

[10] K. Small, "Single wire current sharing paralleling of power supplies," U.S. Patent 4717833 , Jan. 1988.

[11] T. Kohama, T. Ninomiya, M. Shoyama, and F. Ihara, "Dynamic analysis of parallel-module converter system with current balance controllers," in Proc. IEEE Int. Telecom. Energy Conf., 1994, pp. 190-195.

[12] J. Thottuvelil and G. Verghese, "Analysis and control design of paralleled dc/dc converters with current sharing," in Proc. IEEE Appl. Power Electron. Conf., 1997, pp. 638-646.

[13] J. Thottuvelil and G. Verghese, "Stability analysis of paralleled dc-dc converters with active current sharing," in Proc. IEEE Power Electron. Spec. Conf., 1996, pp. 1080-1086.

[14] Y. Panov and M. Jovanović, "Stability and dynamic performance of current-sharing control for paralleled voltage regulator modules," in IEEE Trans. Power Electron., vol. 17, no. 2, pp. 172-179, Mar. 2002.

[15] X. Zhou, P. Xu, and F. C. Lee, "A novel current-sharing control technique for low-voltage high-current voltage regulator module applications," IEEE Trans. Power Electron., vol. 15, no. 6, pp. 1153-1162, Nov. 2000.

[16] R. Erickson and D. Maksimović, Fundamentals of Power Electronics, 2nd ed. Norwell, MA: Kluwer, 2001.

[17] R. D. Middlebrook, "Measurement of loop gain in feedback systems," Int. J. Electron., vol. 38, no. 4, pp. 485-512, 1975. 


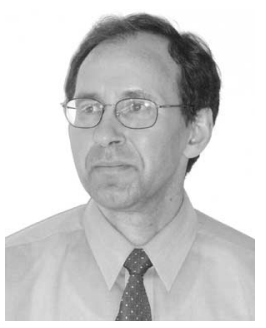

Yuri Panov received the Dipl.-Ing. and Doctoral degrees in electrical engineering from Moscow Aviation Institute, Moscow, Russia, and M.S.E.E. degree from Virginia Polytechnic Institute and State University, Blacksburg.

He is currently a Senior R\&D Staff Member with Delta Products Corporation, Research Triangle Park, NC. His 24-year experience includes dc/ac, ac/dc, and $\mathrm{dc} / \mathrm{dc}$ power conversion, modeling and design of large-scale power systems. His current research is focused on high-power-density dc/dc converters and voltage regulators for the next generation of data-processing equipment.

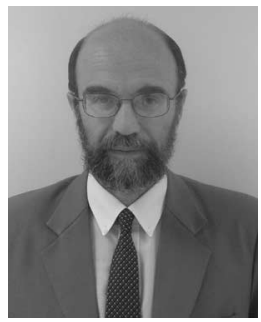

Milan M. Jovanović (F'01) received the Dipl. Ing. degree in electrical engineering from the University of Belgrade, Belgrade, Serbia.

$\mathrm{He}$ is currently the Chief Technology Officer of the Power Systems Business Group, Delta Electronics, Inc., Taipei, Taiwan. 